\title{
Correlação entre intensidade dolorosa e limitação de membro superior em sujeitos com tendinopatia do manguito
}

\section{Correlation between pain intensity and upper limb limitation in subjects with cuff tendinopathy}

Alyssa Conte da Silva ${ }^{1}$, Juliana Falcão Padilha', Jefferson Luiz Brum Marques², Cláudia Mirian de Godoy Marques ${ }^{3}$

${ }^{1}$ Curso de Fisioterapia, Universidade Federal de São Carlos (UFScar) - São Carlos (SP), Brasil.

2Departamento de Engenharia Elétrica e Eletrônica, Universidade Federal de Santa Catarina (UFSC) - Florianópolis (SC), Brasil.

${ }^{3}$ Departamento de Ciências da Saúde, Universidade do Estado de Santa Catarina (UDESC) - Florianópolis (SC), Brasil.

DOI: https://dx.doi.org/10.7322/abcshs.v44i3.1177

\section{RESUMO}

Introdução: A Tendinopatia do Manguito Rotador (TMR) acarreta dor e prejuízo na função do ombro, porém não se sabe se ambas na mesma proporção. Objetivo: Verificar se a intensidade dolorosa se correlaciona com a função do membro superior em sujeitos com TMR. Métodos: Estudo observacional transversal, com 60 indivíduos com TMR. Utilizou-se a Escala Visual Analógica (EVA) para avaliar a dor e o questionário Disabilities of the Arm, Shoulder and Hand Questionnaire (DASH) para função do ombro. Para análise dos dados foi realizada estatística descritiva (médias e desvio-padrão, apresentação numérica e percentual) e aplicada a correlação de Spearman utilizando nível de significância de 5\%. Resultados: A dor média pela EVA foi de 5,02 (moderada). Verificou-se limitação leve (pontuação média de 34,43) nas 30 questões do questionário DASH. Em relação aos módulos opcionais, no módulo destinado a atletas/músicos, não houve limitação dos sujeitos que praticavam este (média de pontuação de 11,82 ). No módulo referente ao trabalho houve limitação leve (média de pontuação de 35,38). Houve correlação baixa entre dor e a função do ombro ( $r s=0,2949 ; p=0,0222$ ). Conclusão: Embora os sujeitos com TMR apresentassem dor moderada, a correlação foi fraca entre dor e função do ombro.

Palavras-chave: ombro; manguito rotador; dor; atividades cotidianas; extremidade superior.

\begin{abstract}
Introduction: Rotator Cuff Tendinopathy (RMR) causes pain and impairment in shoulder function, but it is not known if both in the same proportion. Objective: Verify whether pain intensity correlates with upper limb function in subjects with RMR. Methods: Cross-sectional observational study of 60 individuals with RMR. The Visual Analogue Scale (VAS) was used to assess pain and the Disabilities of the Arm, Shoulder and Hand Questionnaire (DASH) for shoulder function. For data analysis, descriptive statistics (means and standard deviation, numerical and percentage presentation) were performed and Spearman correlation was applied using a significance level of $5 \%$. Results: The average pain by VAS was 5.02 (moderate). Mild limitation (mean score 34.43) was found in the 30 questions of the DASH questionnaire. Regarding the optional modules, in the module intended for athletes/musicians, there was no limitation of the subjects who practiced this (average score of 11.82). In the work module there was a slight limitation (average score 35.38). There was a low correlation between pain and shoulder function ( $r s=0.2949 ; p=0.0222)$. Conclusion: Although subjects with RMR had moderate pain, the correlation was weak between pain and shoulder function.
\end{abstract}

Keywords: shoulder; rotator cuff; pain; activities of daily living; upper extremity. 


\section{INTRODUÇÃO}

A dor no ombro é a terceira queixa musculoesquelética mais comum sendo mais recorrente em adultos e sua frequência aumentando com o passar da idade ${ }^{1,2}$. Nesse contexto, destacam-se as tendinopatias, em especial, as Tendinopatias do Manguito Rotador (TMR) ${ }^{3}$, que incluem ruptura parcial e/ou lesão completa dos tendões supraespinhoso, infraespinhoso e/ou subescapular, podendo ainda estar associada com tendinopatia do tendão da cabeça longa do bíceps ${ }^{4}$, bem como processos inflamatórios destas estruturas.

A TMR pode acometer indivíduos em qualquer faixa etária, tendo a sua incidência aumentada com o envelhecimento e a ocupação laborativa ou recreativa ${ }^{3}$. Geralmente a TMR pode levar ao comprometimento da função do ombro em vários graus, principalmente devido à dor ${ }^{5}$. Uma das principais formas de verificar a dor no ombro no ambiente clínico é por meio da Escala Visual Analógica (EVA), embora esta seja uma avaliação subjetiva da dor, é de fácil uso, assim como amplamente utilizada em diversas doenças musculoesqueléticas ${ }^{6,7}$.

Devido à dor imposta pela lesão, as atividades de vida diária dos sujeitos que apresentam TMR podem ficar muito prejudicadas. Tal fato acarreta diminuição da função do membro superior acometido, baixa qualidade de vida, diminuição da qualidade do sono, absenteísmo no trabalho, dentre outros prejuízos ${ }^{8}$.

Uma forma de se avaliar a funcionalidade do membro superior se faz por meio de questionários funcionais, como por exemplo, o Disabilities of the Arm, Shoulder and Hand Questionnaire (DASH). O questionário DASH é um dos poucos traduzidos e validados no Brasil que avalia os sintomas, as funções físicas, sociais e psicológicas do indivíduo que englobam o braço, ombro e mão. Além disso, este questionário aborda funções diárias tais como varrer, pentear o cabelo, colocar objetos em cima de prateleiras, entre outras, constituindo atividades comuns e presentes no dia a dia ${ }^{9,10}$.

No entanto, embora indivíduos com TMR apresentem dor que possa influenciar suas atividades funcionais, são poucos os estudos que verificam se o nível de dor afeta da mesma forma a função do ombro. Em razão disso, o estudo teve por objetivo verificar se a intensidade dolorosa se correlaciona com a função do membro superior em sujeitos com TMR. A hipótese do estudo é a de que quanto mais alta a intensidade dolorosa, maior a correlação com os prejuízos funcionais do membro superior, uma vez indivíduos com TMR tendem a apresentarem redução da função do ombro ${ }^{11,12}$, impactando negativamente sobre as atividades funcionais ${ }^{13,14}$.

\section{MÉTODOS}

Trata-se de um estudo observacional de caráter transversal, com abordagem quantitativa, realizado na Clínica Escola de Fisioterapia da Universidade do Estado de Santa Catarina (UDESC) na cidade de Florianópolis (SC) e na Clínica Fisioativa na cidade de Santa Maria (RS). A pesquisa obteve aprovação do Comitê de Ética e Pesquisa em Seres Humanos da UDESC sob-registro de CAAE no 37088014000000118 . Todos os sujeitos assinaram o Termo de Consentimento Livre e Esclarecido.

O processo de seleção dos participantes da pesquisa foi de maneira intencional. Inicialmente aplicou-se uma ficha de anamnese para coletar dados pertinentes à pesquisa, tais como idade, sexo, tempo de dor no ombro, entre outros. Em seguida, realizou-se uma avaliação cinético-funcional por uma fisioterapeuta para comprovar a lesão. A avaliação consistiu em 5 testes clínicos, onde os indivíduos deveriam apresentar resultado positivo no mínimo em 3 destes, indicando assim sinais de lesão do manguito rotador ${ }^{15}$. Os testes foram:

1. Teste de Hawkins positivo;

2. Teste de Neer positivo;

3. dor durante a elevação ativa menor que 60 graus no plano de escápula;

4. Teste de Jobe positivo (caneca vazia);

5. dor ou fraqueza com rotação externa do ombro resistida com o braço ao lado do corpo.

Após, a dor foi avaliada por meio da EVA. Esta escala consiste em uma linha horizontal de $10 \mathrm{~cm}$ onde consta o número 0 (zero) marcado na extremidade esquerda da linha correspondendo a nenhuma dor e o número 10 (dez) marcado na extremidade direita da mesma linha correspondendo à dor máxima ${ }^{16}$. Assim, os indivíduos marcavam um traço vertical na linha para demarcar o nível de dor e o avaliador fazia a mensuração com uma régua do marco 0 até o traço assinalado pelos sujeitos.

Por fim, foi aplicado o questionário DASH. O questionário é composto por 30 questões que avaliam os sintomas e as funções físicas, sociais e psicológicas. Cada questão tem 5 possibilidades de resposta que variam entre não haver dificuldade e não conseguir realizar a atividade com pontuação de 1 a 5 . A pontuação final do questionário varia de 0 a 100, sendo 1-20 (sem limitação), 21-40 (limitação leve), 41-60 (limitação moderada), 61-80 (limitação severa) e 81-100 (limitação muito severa). Além disso, há dois módulos com itens opcionais: um para atletas e/ou músicos e outro para os trabalhadores. A pontuação do questionário é calculada por meio da aplicação de fórmulas estabelecidas, uma delas usada para analisar as primeiras 30 questões e outra usada separadamente para os módulos opcionais ${ }^{9,10}$.

Foram adotados como critérios de inclusão: diagnóstico clínico de TMR; homens e mulheres; faixa etária entre 20 e 70 anos de idade; apresentar dor há pelo menos seis meses; submeter-se à avaliação cinético-funcional; não estar usando anti-inflamatório por tempo mínimo de um mês; não estar em tratamento fisioterapêutico e apresentar dor de intensidade maior que três EVA ${ }^{16}$.

Para os critérios de exclusão empregou-se: sujeitos com sinais clínicos de lesão completa do manguito rotador ("Drop Arm Test" 
positivo); que tivessem sido submetidos a intervenção cirúrgica nos ombros; queixa dolorosa na coluna vertebral (região torácica); histórico de cirurgia ou trauma na coluna vertebral, gestantes; histórico de câncer; doença neurológica e deficientes visuais e/ou auditivos Foi realizada análise descritiva com média e desvio padrão, apresentação numérica e percentual. Para realizar a análise de correlação entre dor e o DASH, a normalidade das variáveis foi testada por meio do teste de Shapiro Wilk. Uma vez que a variável "dor" não apresentou distribuição normal ( $\mathrm{p}=0,0019)$, foi aplicada o coeficiente de correlação de Spearman (rs) utilizando nível de significância de 5\%. A força do coeficiente levou em consideração os seguintes valores: entre 0,00 e 0,25 correlação pequena; entre 0,26 e 0,49 correlação baixa; entre 0,50 e 0,69 correlação moderada; entre 0,70 e 0,89 correlação alta; e entre 0,90 e 1,00 correlação muito alta ${ }^{17}$. A análise estatística foi realizada por meio do software $R$ versão 3.4.1.

\section{RESULTADOS}

A amostra foi composta por 60 indivíduos sendo 41 do sexo feminino e 19 do sexo masculino. Quanto à prática de exercício físico, 58,33\% não praticavam nenhum exercício físico. As características da amostra encontram-se na Tabela 1.

O ombro que apresentou maior acometimento foi o direito, correspondendo a 66,67\%. Em relação à dor, o tempo médio que os sujeitos apresentaram este sintoma foi de 3,76 anos. Já o nível de dor verificado pela EVA foi de 5,02 cm (moderado).

Os dados gerais do questionário DASH apresentam-se na Tabela 2. Já os resultados referentes à distribuição por sub-limitação encontram-se na Tabela 3.

Em relação à dor e a função do ombro (DASH), obteve-se uma correlação baixa ( $r s=0,2949$ ), com $p=0,0222$. O comportamento conjunto destas variáveis está apresentado na Figura 1.

\section{DISCUSSÃO}

O presente estudo evidenciou de forma geral que os sujeitos apresentaram dor moderada e houve correlação, porém, fraca, entre dor e limitação do ombro. Tal fato foi inesperado, pois se acreditava que a dor fosse repercutir da mesma maneira sobre as atividades funcionais, fato este que não ocorreu, refutando a hipótese do estudo. Assim, sugere-se que este resultado possa ser justificado pelo fato de os sujeitos apresentarem dor crônica local (região dos ombros).

A dor crônica é caracterizada por uma sensibilização central refletindo uma dor que em grande parte é impulsionada pela sensibilização no sistema nervoso central e deficiência descendente na modulação da dor ${ }^{18}$. No entanto, existem determinadas condições de dor crônica que são mais localizadas em articulações e nem sempre a sensibilização central está presente, como por exemplo, em indivíduos com dor no ombro ${ }^{19}$. Dessa forma, os indivíduos desse estudo embora apresentassem dor crônica (média de 3,76

Tabela 1: Características da amostra.

\begin{tabular}{l|c|}
\hline Variáveis & $\begin{array}{c}\text { Sujeitos }(\mathbf{n}=60) \\
\text { Média } \pm \text { desvio padrão }\end{array}$ \\
\hline Idade (anos) & $45,27 \pm 14,17$ \\
\hline Altura $(\mathrm{m})$ & $1,66 \pm 0,09$ \\
\hline Peso $(\mathrm{kg})$ & $74,23 \pm 14,63$ \\
\hline
\end{tabular}

Tabela 2: Classificação geral do questionário DASH (Disabilities of the Arm, Shoulder and Hand).

\begin{tabular}{|l|c|c|c|}
\hline Questionário DASH & $\begin{array}{c}\mathbf{3 0} \\
\text { Questões }\end{array}$ & $\begin{array}{c}\text { Módulo 1 } \\
\text { Exercício Físico }\end{array}$ & $\begin{array}{c}\text { Módulo 2 } \\
\text { Trabalho }\end{array}$ \\
\hline Pontuação & 34,43 & 11,82 & 35,38 \\
\hline Limitação & Leve & Sem Limitação & Leve \\
\hline
\end{tabular}

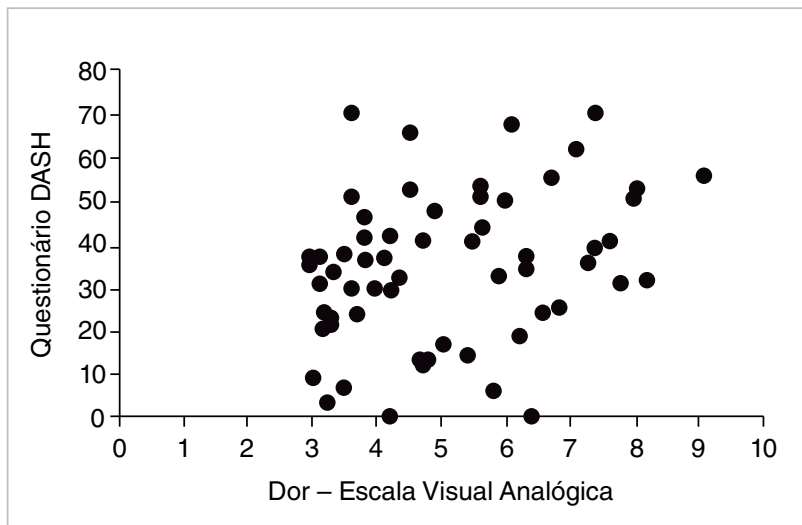

Figura 1: Dispersão entre a escala de dor e o questionário DASH (Disabilities of the Arm, Shoulder and Hand).

Tabela 3: Classificação do questionário DASH (Disabilities of the Arm, Shoulder and Hand) sobre a sub-limitação.

\begin{tabular}{|c|c|c|c|}
\hline Questionário DASH & 30 Questões & $\begin{array}{c}\text { Módulo } 1 \\
\text { Exercício Físico }\end{array}$ & $\begin{array}{l}\text { Módulo } 2 \\
\text { Trabalho }\end{array}$ \\
\hline Sem limitação & $20 \%(n=12)$ & $25 \%(n=15)$ & $31,68 \%(n=19)$ \\
\hline Limitação leve & $46,67 \%(n=28)$ & $10 \%(n=6)$ & $28,33 \%(n=17)$ \\
\hline Limitação moderada & $25 \%(n=15)$ & $1,67 \%(n=1)$ & $18,33 \%(n=11)$ \\
\hline Limitação severa & $8,33 \%(n=5)$ & $1,67 \%(n=1)$ & $18,33 \%(n=11)$ \\
\hline Muito severa & $0 \%(n=0)$ & $3,33 \%(n=2)$ & $3,33 \%(n=2)$ \\
\hline Não responderam a este item & - & $58,33 \%(n=35)$ & - \\
\hline
\end{tabular}

*Não praticavam exercício físico e/ou esporte 
anos) esta foi localizada, não ocorrendo uma sinalização nociceptiva reforçada, o que possivelmente permitiu que os sujeitos realizassem as tarefas funcionais sem grandes limitações e queixas dolorosas.

Além disso, outro aspecto verificado nessa pesquisa é em relação aos indivíduos que praticam algum exercício físico e/ou esporte, onde estes não apresentaram limitação da função do ombro. Pesquisas mostram que tanto um aumento quanto uma diminuição no limiar da dor podem ocorrer em pacientes com dor crônica quando envolvidos em diferentes tipos de exercícios ${ }^{20,21}$. Diante disso, como a presente população apresentava dor crônica com queixa localizada, aparentemente sem sensibilização central dominante, o exercício pode ter auxiliado esses sujeitos.

Esse possível benefício do exercício pode ter ocorrido em razão de que parece que há um mecanismo de inibição da dor endógena que pode estar ativo durante o exercício, o que resulta em limiar de dor aumentado tanto durante quanto após este $e^{20,21,22}$. Com isso, os sujeitos tornam-se mais toleráveis a dor, não afetando com severidade sua função.

No entanto, vale ressaltar que este efeito parece ser dependente do paciente e do tipo de exercício (treinamento aeróbio geral ou treinamento de força, por exemplo $)^{20,21}$. Ainda, se os exercícios são realizados envolvendo diferentes partes do corpo isso pode resultar em alívio da dor nestes pacientes, reduzindo a sensibilidade à dor nos tecidos afetados. Por outro lado, foi visto que exercícios específicos que envolvam partes do corpo dolorosas nem sempre ativam mecanismos inibitórios segmentares ou multissegmentares da dor em pacientes com queixas de dor muscular nos ombros, por exemplo ${ }^{20,21,22}$. Este aspecto quanto ao tipo de exercício, bem como se esses foram gerais ou locais não foram abordados pela pesquisa, constituindo uma limitação do estudo.

Em relação aos sujeitos da amostra, verificou-se que os indivíduos apresentaram média de idade de 45,27 anos, sendo a maioria mulheres. Nesse contexto, há estudos mostrando que as queixas tendem a aumentar com a idade ${ }^{23} \mathrm{e}$ as mulheres são mais propensas a apresentarem distúrbios musculoesqueléticos ${ }^{24}$, bem como tendem a apresentar limiar doloroso menor que dos homens ${ }^{25}$.

Demais estudos em sujeitos com TMR também fizeram uso do DASH para avaliar a função do ombro mostrando que este questionário pode ser utilizado tanto para uma avaliação inicial, quanto para reavaliação da função do ombro após intervenções e/ ou após um período de tratamento ${ }^{26,27}$. Da mesma forma, outros estudos utilizaram a EVA para quantificar a dor em indivíduos com $\mathrm{TMR}^{28}$. Logo, tanto o uso do DASH quanto da EVA se faz totalmente viável na prática clínica e propõe-se que deva ser encorajada sua utilização, uma vez que ambos são de fácil aplicação e entendimento, tornando possível acompanhar o paciente de forma mais objetiva no processo de reabilitação.

Entretanto, embora a aplicabilidade de ambos os instrumentos na clínica seja relevante, é importante destacar que nem sempre o fisioterapeuta poderá inferir que o nível de dor traz o mesmo prejuízo na função do ombro, pois como foi verificado nessa pesquisa, embora os sujeitos apresentassem dor considerada moderada, o grau de limitação nas funções foi considerado leve.

Devem-se ressaltar algumas limitações que fizeram parte da pesquisa. A primeira é referente à ausência de um cálculo amostral, o que não permite verificar a validade externa do estudo para populações com características diferentes. A segunda limitação é referente à amostra ser constituída apenas por sujeitos com dor crônica com ampla variação de idade, além da utilização somente de um instrumento para verificar a dor, bem como a função do ombro, não sabendo se com uso de outra escala e/ou outro questionário para função esses resultados seriam diferentes. Por último, a ausência de um exame de imagem para diagnóstico associado aos testes clínicos, o que poderia ter proporcionado maior acurácia ao diagnóstico.

Em conclusão, foi possível verificar que os indivíduos com TMR apresentaram dor moderada e verificou-se limitação leve nas atividades funcionais e no trabalho, sem apresentar limitação nos indivíduos que praticavam algum exercício físico. Além disso, houve correlação, porém, esta correlação foi fraca entre dor e função do ombro avaliado pelo questionário DASH. Portanto, o nível de dor não se refletiu na mesma proporção na função do ombro. Sugere-se para estudos posteriores a inclusão de sujeitos com dor aguda e/ou a subdivisão de grupos conforme o tempo de dor crônica, a adição de demais instrumentos que avaliem dor e aspectos funcionais do ombro, bem como investigar o tipo de exercício que os sujeitos praticam (se aeróbio ou de força), assim como sua periodização.

\section{REFERÊNCIAS}

1. Teunis T, Lubberts B, Reilly BT, Ring D. A systematic review and pooled analysis of the prevalence of rotator cuff disease with increasing age. J Shoulder Elbow Surg. 2014;23(12):1913-21. http://dx.doi.org/10.1016/j.jse.2014.08.001

2. Motta GR, Amaral MV, Rezende E, Pitta R, Vieira TC, Duarte ME, et al. Evidence of genetic variations associated with rotator cuff disease. J Shoulder Elbow Surg. 2014;23(2):227-35. http://dx.doi.org/10.1016/j.jse.2013.07.053
3. Saggini R, Cavezza T, Di Pancrazio L, Pisciella V, Saladino G, Zuccaro MC, et al. Treatment of lesions of the rotator cuff. J Biol Regul Homeost Agents. 2010;24(4):453-9.

4. Lasbleiz S, Quintero N, Ea K, Petrover D, Aout M, Laredo JD, et al. Diagnostic value of clinical tests for degenerative rotator cuff disease in medical practice. Ann Phys Rehabil Med. 2014;57(4):228-43

http://dx.doi.org/10.1016/j.rehab.2014.04.001 
5. Braman JP, Zhao KD, Lawrence RL, Harrison AK, Ludewig PM. Shoulder impingement revisited: evolution of diagnostic understanding in orthopedic surgery and physical therapy. Med Biol Eng Comput. 2014;52(3):211-9. http://dx.doi.org/10.1007/s11517-013-1074-1

6. Esculier JF, Bouyer LJ, Dubois B, Fremont P, Moore L, McFadyen $\mathrm{B}$, et al. Is combining gait retraining or an exercise programme with education better than education alone in treating runners with patellofemoral pain? A randomised clinical trial. $\mathrm{Br} \mathrm{J}$ Sports Med. 2018;52(10):659-66.

http://dx.doi.org/10.1136/bjsports-2016-096988

7. Camargo PR, Alburquerque-Sendín F, Avila MA, Haik MN, Vieira A, Salvini TF. Effects of Stretching and Strengthening Exercises, With and Without Manual Therapy, on Scapular Kinematics, Function, and Pain in Individuals With Shoulder Impingement: A Randomized Controlled Trial. J Orthop Sports Phys Ther. 2015;45(12):984-97. http://dx.doi.org/10.2519/jospt.2015.5939

8. Factor D, Dale B. Current concepts of rotator cuff tendinopathy. Int J Sports Phys. Ther. 2014;9(2):274-88.

9. Orfale AG, Araújo PMP, Ferraz MB, Natour J. Translation into Brazilian Portuguese, cultural adaptation and evaluation of the reliability of the Disabilities of the Arm, Shoulder and Hand Questionnaire. Braz J Med Biol Res. 2005;38(2):293-302. http://dx.doi.org/10.1590/S0100-879X2005000200018

10. Pinho AB, Borghesan FHP, Lotufo MN, Allet MA. Avaliação dos tratamentos cirúrgicos das sequelas de hanseníase pelas escalas Salsa e Dash. Rev Bras Ortop. 2014;49(3):292-6. http://dx.doi.org/10.1016/j.rboe.2014.04.004

11. Shin KM. Partial-thickness rotator cuff tears. Korean J Pain. 2011;24(2):69-73. https://doi.org/10.3344/kjp.2011.24.2.69

12. Braman JP, Zhao KD, Lawrence RL, Harrison AK, Ludewig PM. Shoulder impingement revisited: evolution of diagnostic understanding in orthopedic surgery and physical therapy. Med Biol Eng Comput. 2014;52(3):211-9.

https://doi.org/10.1007/s11517-013-1074-1

13. Macdermid J, Ramos J, Drosdowech D, Faber K, Patterson S. The impact of rotator cuff pathology on isometric and isokinetic strength, function, and quality of life. J Shoulder Elbow Surg. 2004;13(6):593-8.

https://doi.org/10.1016/S1058274604001247

14. Tekeoglu I, Ediz L, Hiz O, Toprak M, Yazmalar L, Karaaslan G. The relationship between shoulder impingement syndrome and sleep quality. Eur Rev Med Pharmacol Sci. 2013;17(3):370-4.

15. Michener LA, Walsworth MK, Doukas WC, Murphy KP. Reliability and diagnostic accuracy of 5 physical examination tests and combination of tests for subacromial impingement. Arch Phys Med Rehabil. 2009;90(11):1898-903.

http://dx.doi.org/10.1016/j.apmr.2009.05.015

16. Tashijan RZ, Deloach J, Porucznik CA, Powell AP. Minimal clinically important differences (MCID) and patient acceptable symptomatic state (PASS) for visual analog scales (VAS) measuring pain in patients treated for rotator cuff disease. J Shoulder Elbow Surg. 2009;18(6):927-32 http://dx.doi.org/10.1016/j.jse.2009.03.021

17. Munro BH. Statisticals methods for health care research. 4. ed. Philadelphia: Lippincott; 2001.

18. Ashmawi HA, Freire GMG. Sensibilização periférica e central. Rev Dor. 2016:17(Suppl 1):S31-4. http://dx.doi.org/10.5935/1806-0013.20160044

19. Meeus M, Nijs J, Van Wilgen P, Noten S, Huijnen I. Moving on to movement in patients with chronic joint pain. Pain: Clin Updates. 2016;24(1):1-8.

20. Meeus M, Roussel NA, Truijen S, Nijs J. Reduced pressure pain thresholds in response to exercise in chronic fatigue syndrome but not in chronic low back pain: an experimental study. J Rehabil Med. 2010;42(9):884-90. http://dx.doi.org/10.2340/16501977-0595

21. Meeus M, Hermans L, Ickmans K, Struyf F, Van Cauwenbergh D, Bronckaerts $\mathrm{L}$, et al. Endogenous pain modulation in response to exercise in patients with rheumatoid arthritis, patients with chronic fatigue syndrome and comorbid fibromyalgia, and healthy control a double-blind randomized controlled trial. Pain Pract. 2015;15(2):98-106.

http://dx.doi.org/10.1111/papr.12181

22. Burrows NJ, Booth J, Sturnieks DL, Barry BK. Acute resistance exercise and pressure pain sensitivity in knee osteoarthritis: a randomised crossover trial. Osteoarthritis Cartilage. 2014;22(3):407-14

http://dx.doi.org/10.1016/j.joca.2013.12.023

23. Lautenbacher S, Peters JH, Heesen M, Scheel J, Kunz M. Age changes in pain perception: A systematic-review and metaanalysis of age effects on pain and tolerance thresholds. Neurosci Biobehav Rev. 2017;75:104-13.

http://dx.doi.org/10.1016/j.neubiorev.2017.01.039

24. Almeida CGSTG, Fernandes RCP. Distúrbios musculoesqueléticos em extremidades superiores distais entre homens e mulheres: resultados de estudo na indústria. Rev Bras Saude Ocup. 2017;42:e3. http://dx.doi.org/10.1590/2317-6369000125515

25. Pieretti S, Di Giannuario A, Di Giovannandrea R, Marzoli F, Piccaro $G$, Minosi $P$, et al. Gender differences in pain and its relief. Ann Ist Super Sanita. 2016;52(2):184-9. http://dx.doi.org/10.4415/ANN 160209

26. Desjardins-Charbonneau A, Roy JS, Dionne CE, Desmeules F. The efficacy of taping for rotator cuff tendinopathy: a systematic review and meta-analysis. Int J Sports Phys Ther. 2015;10(4):420-33.

27. Amrood MN, AsI HS, Salariyeh M, Safari MB. Pain, disability and sleep quality in patients with rotator cuff tendinopathy and concurrent myofascial pain. Shafa Ortho J. 2016;3(2):e4481. http://dx.doi.org/10.17795/soj-4481

28. Bury J, West M, Chamorro-Moriana G, Littlewood C. Effectiveness of scapula-focused approaches in patients with rotator cuff related shoulder pain: A systematic review and meta-analysis. Man Ther. 2016;25:35-42.

http://dx.doi.org/10.1016/j.math.2016.05.337 